\title{
A monitoring device for pressurised-air-driven diaphragm-based artificial heart assist devices
}

\author{
F P Hoeben, F F M de Mul, H S D Stokkink, M H Koelink and \\ J Greve \\ University of Twente, Department of Applied Physics, PO Box 217, 7500 AE \\ Enschede, The Netherlands
}

Received 8 April 1991, in final form 17 July 1991

\begin{abstract}
A non-invasive device has been developed to monitor the diaphragm position and the blood flow in artificial heart assist devices equipped with a pressurisedair-driven diaphragm. Light scattering from the diaphragm is used as a mechanism for measuring. Information about the position of several points of the diaphragm can be obtained. The completely empty or filled situation can be detected and used for control purposes. Flow data can be extracted and bending characteristics of the diaphragm during operation can be studied.
\end{abstract}

\section{Introduction}

A commonly used type of artificial heart assist device consists of a morc-or-less globular vessel (diameter $\approx 5 \mathrm{~cm}$ ), made out of synthetic material and divided into two parts by a flexible membrane (diaphragm). One part of the device is filled with the blood to be transported, the other part is filled with pressurised air under variable pressure (figure 1). The blood side of the device has a blood inlet and outlet, each equipped with a proper one-way valve. By varying the gas pressure the diaphragm will move from one wall to the other and pumping of the blood is obtained (Wurzel et al 1988).

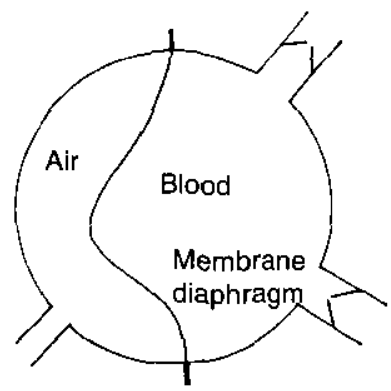

Figure 1. Schematic drawing of an artificial heart assist device with air-pressure control.

To monitor the performance of artificial heart assist devices it is necessary to obtain information about the position of the diaphragm. This is important in order to control the pumping process, to study the bending characteristics of the diaphragm and to obtain quantitative data about the flow. 
Methods now available for the control of the pumping process make use of external measurement of the air pressure or the blood flow. Complete filling or emptying of the air chamber can be detected as changes in the air pressure. The disadvantage of this system is the inefficiency of using the pumping power itself to detect the extremes of the pumping process, and the sensitivity of the measurements to changes in the volume and the temperature of the device during the process. Moreover, since normally long and thin air tubes are used, some time delay may occur in the detection of the pressure changes.

In addition, magnetic sensors, in which a small magnet is attached to the diaphragm, and an inductive coil placed outside the device, are used. The attachment of the magnet to the diaphragm may, however, influence the bending process; changes in the shape of the diaphragm during operation also cause changes in the direction of the magnetic axis and therefore in the inductance signal strength.

Electromagnetic devices measuring flow utilising the Hall effect, attached to the inlet or outlet pipes or to arteries, often introduce problems with the attachment of the magnets or the electric contacts.

In the search for an independent measuring device a number of restrictions have to be considered. Physical contact with the blood, which would introduce blood compatibility problems, has to be avoided. The device should not disturb the blood pumping process. When used for control purposes the instrument should operate completely independently of the driving (air pressure) process. Other body functions should also not be influenced, so large electrical currents should be avoided. The dimensions of ủe insirumenit shioùiu be chōseñ sô small that a simple fixation on the artificial heart or even implantation together with the artificial heart would bccome possible. Finally, low power consumption is necessary.

The restrictions mentioned above suggest an optical solution. This solution was applied in the instrument described in the following sections.

\section{Description of the instrument}

The instrument developed consists of a probe containing one or several light sources and photodiodes, and is attached at the outside of the wall at the air side of the heart assist device. Light scattering by the diaphragm through the wall is used as a measure for the position of the diaphragm. The only conditions are adequate transparency of the wall, and a sufficient scattering efficiency of the diaphragm or the underlying blood for the light colour chosen.

The diaphragm of the type of heart assist devices under consideration, made out of synthetic material (e.g. polyurethane), is designed in such a way that damage due to mechanical stress is minimised. This is realised by making the diaphragm as flexible as possible. For this reason the diaphragm bends in a very irregular way. (Because of this effect the use of magnetic detection in which a small magnet is attached to the diaphragm is seriously hampered). Determination of the position of a single point on the diaphragm (preferably the centre point) is thus not sufficient. More points on the diaphragm should be taken into consideration. The signals from the individual points may then be added to provide an averaged position of the diaphragm or they may be considered individually to provide insight into the way the diaphragm bends. The positions of chosen points on the diaphragm are obtained by measuring the intensity of light, emitted by sources outside the artificial heart, scattered by the diaphragm and detected by one or several photodiodes 
placed near the sources. The resulting intensity is approximately inversely proportional to the square of the distance between the diaphragm and the detector, which dependence can easily be calibrated before clinical application of the instrument. The exact relation depends on geometrical and experimental factors, like the divergence of the light source.

The actual probe consists of a ring of six sets of an LED source and two photodiodes with a seventh pair in the centre (figure 2). The LEDS emit at $650 \mathrm{~nm}$ with a reasonably small divergence (about $10^{\circ}$ ). This small divergence is only necessary in case all diodes have to be read out separately. In one model the LEDs and diodes are mounted on a flexible printed circuit board containing the electrical printed circuit. Another model has the LEDs and diodes embedded in a thin flexible disc of transparent silicone material. For implantation purposes other body-friendly material will do as well. These probes can easily be glued to the curved rear of the device, or incorporated in the material of the back wall itself. The current probe is about $25 \mathrm{~mm}$ in diameter and $5 \mathrm{~mm}$ high.
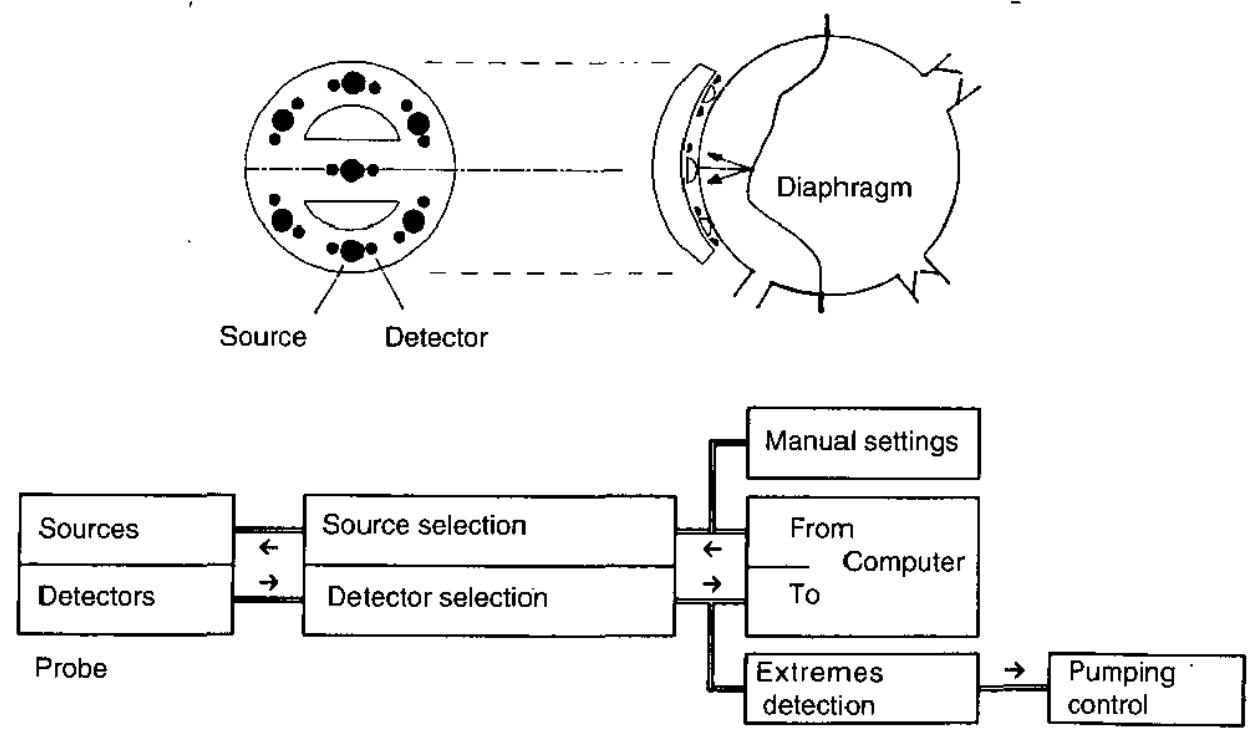

Figure 2. The optical blood flow probe, to be attached to the artificial heart assist device, with the signal processing unit.

To obtain a high scattering efficiency the diaphragm may be coated with a flexible white material like Decron. Another solution is the use of a sufficiently transparent diaphragm; light will then be scattered by the first layer of blood cells at the other side of the diaphragm. A diaphragm showing light reflection rather than scattering cannot be used for the study of the motional behaviour of the diaphragm, since reflection does not provide distance-dependent information. On the other hand, if one is primarily interested in the extremes of diaphragm position only (completely empty or filled situation) a purely reflecting diaphragm will work properly, since in this case, as a result of the irregular bending of the diaphragm during each cycle, only at the extreme positions will the diaphragm allow specular reflection from the source in the backward direction to the detector. Then, only with the diaphragm at one of the two extreme positions will a response at the 
detector be observed. In this case no distance-dependent information is needed. One source and one detector, placed at a position on the symmetry axis of the heart assist device, will be sufficient: the probe can then be made very small. Such a probe has been constructed.

The signal processing unit performs lock-in amplification of the photodiode signals with $10 \mathrm{kHz}$ pulsing of the LEDs. In this way most of the electrical noise and environmental light noise is suppressed. The unit also selects the particular light source(s) and detector(s) to be used. The unit can be operated in two ways. Firstly, the probe can be used with all sources parallel and all detector signals added. In this way a spatially averaged position of the diaphragm is obtained. Moreover, the signal processing unit is equipped with a detection facility of predefined signal levels, which signals can be used for control purposes of the pumping process. Secondly, every source/detectors set can be monitored separately (subsequently for the probe of figure 2 this means $7 \times 14=98$ combinations). After additional processing using a computer this may provide information about the actual position of the individual (seven) points of the diaphragm during the pumping process. The specific characteristics of each source (light output relative to current) and detector (sensitivity) can be measured and stored before assembling, to provide data for online signal normalisation during operation.

If the LEDs arc inadequate with respect to intensity or divergence, they can be replaced by small semiconductor lasers.

Befưre clinical use the instruñnent has to be calibratcd in combination with the particular heart assist device. This can be done using an external volumetric standard, as will be described in the following section.

Ageing of the device, possibly leading to changes in the calibration, can be a problem. The gravity of this problem will depend on the construction of the device and on potential degradation of the scattering properties of the diaphragm. Some further research will be necessary. However, when application is restricted merely to measuring the extremal positions of the diaphragm (e.g. for control purposes), only relative signal changes are important, and the electronics can be made to deal with those quite easily.

\section{Test measurements with the instrument: discussion of measurements}

For the extraction of flow data the relation between the light intensity and the flow is necessary. The light intensity depends on the distances between the chosen puints on the diaphragm and the probe, and the volume of the blood side of the device is also related to the position of the diaphragm. From a pragmatic point of view this relation can easily be obtained using an external volumetric calibration standard.

In figure 3 some typical results obtained with the instrument are shown. The measurements were done using a $60 \mathrm{~cm}^{3}$ polyurethane ventricle. This ventricle has a single-layer diaphragm which is covered with white Decron to improve isotropic scattering. This layer was attached during the production process and was shown to have no noticeable influence on the bending characteristics. The ventricle was used with a full stroke of $62 \mathrm{~cm}^{3}$ (more than $60 \mathrm{~cm}^{3}$ because of the elasticity of the ventricle). For the external calibration of the volume of the blood side of the device, during each cycle the blood was pumped from a storage vessel through the device into a vertical tube provided with a level sensor. An external computer attached to 
the probe read out the signals and performed the calculation of flow data. In figure $3(b)$ a stroke volume of $30 \mathrm{~cm}^{3}$ was used for comparison. The probe has been glued to the air chamber of the ventricle. The LEDS were used parallel; the photodiode signals were added together.
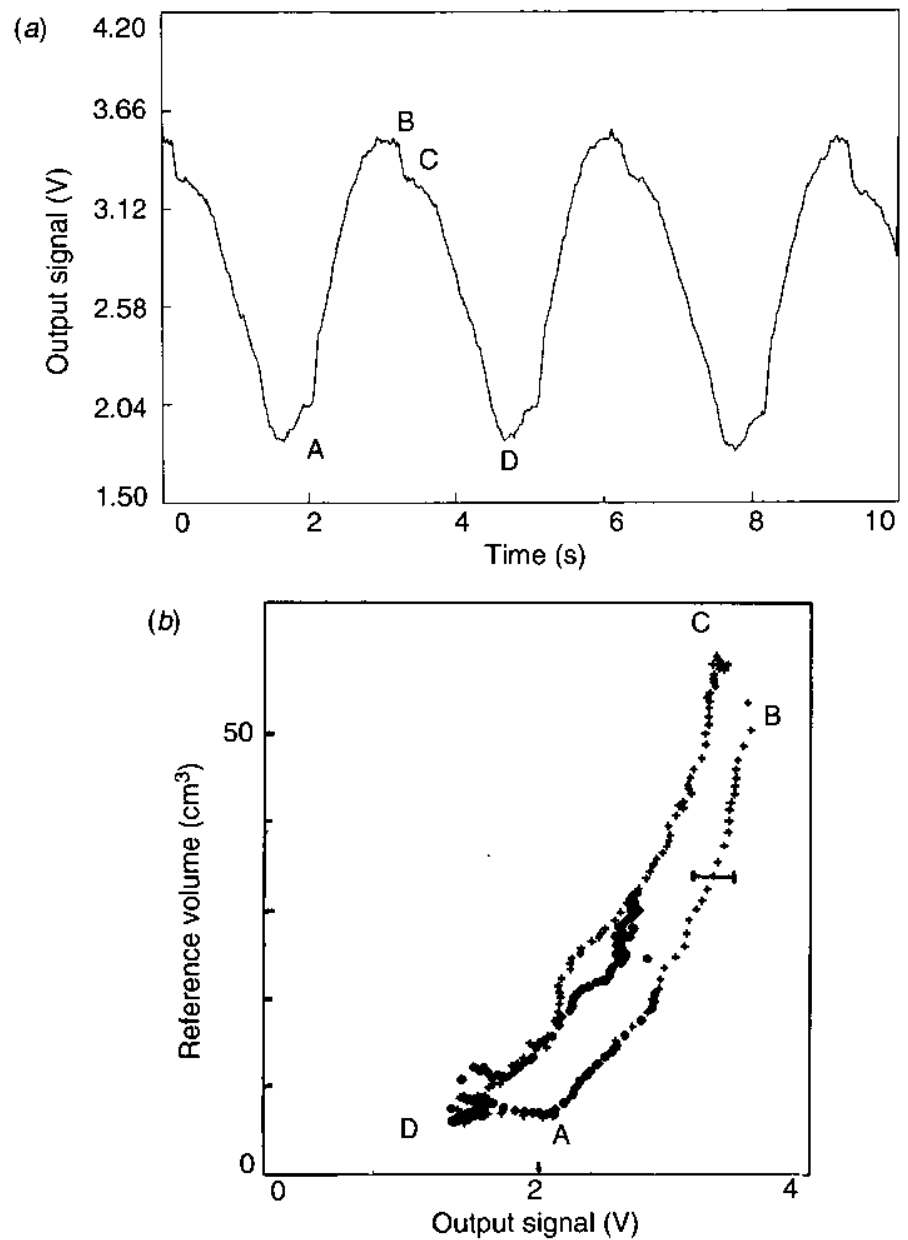

Figure 3. Signal from the probe while pumping blood: (a) as a function of time (stroke volume $62 \mathrm{~cm}^{3}$ ); (b) as a function of volume using an external calibration; stroke volumes of $62 \mathrm{~cm}^{3}(+)$ and $30 \mathrm{~cm}^{3}(0)$; error bar is uncertainty over 2000 cycles due to bending (see text).

In figure $3(a)$ the light intensity output signal of the diodes is shown as a function of time. At point $A$ the (air) pumping process starts (systolic phase), while in $B$ the pressure is released. From $B$ to $C$ the ventricle relaxes elastically, while from $C$ to $D$ the relaxed device is filled with blood (diastolic phase) and the diaphragm moves backwards towards the detectors. Point $\mathrm{D}$ is lower than point $\mathrm{A}$ due to the elastic expansion of the device caused by the cumulative momentum pressure of the blood in the device and the circulation system. Our system enables detection of those features for optimisation of the efficiency of the pumping process in which point $C$ should approach $B$ and $A$ should approach $D$. The figure shows a remarkable reproducibility, sufficient for a precise setting of control levels indicating 
the (almost) completely full or empty situation. Figure 3(b) shows the output signal plotted against the external volumetric calibration standard, for two stroke volumes of $62 \mathrm{~cm}^{3}$ and $30 \mathrm{~cm}^{3}$, respectively. The figure shows a hysteresis curve for each stroke volume. The hysteresis originates from the elasticity of the ventricle. In the systolic phase, when pressure is high, there is some deformation of the ventricle which causes the ventricle to contain more blood at the same sensor signal level than in the diastolic phase. Points A, B, C and D have the same meaning as in figure 3(a). The curves show good agreement during the systolic phase. During the diastolic phase the agreement is less good. This can be caused by differences in the momentum of the blood at different stroke volumes, which will lead to different elastic expansion. The error bar represents uncertainty over $2000 \mathrm{cycles}$, due to irregularities in the bending process. However, the error in the extreme points of the curve (representing the pumped volume) are much smaller: of the order of the symbol size; the uncertainty in the flow over all cycles is therefore very low.

With this instrument the blood flow of a similar heart assist device, externally assisting the heart of a healthy calf, was monitored. Similar results to those shown in figure $3(a)$ could be obtained.

\section{Conclusions}

The instrument described in the foregoing sections has been shown to be able to control the pumping process of pressurised-air-driven artificial heart assist devices equipped with a flexible diaphragm. It is also able to provide flow data during the pumping process. The main feature of the device is the avoidance of blood contact and the absence of influence on the air pumping system.

\section{Acknowledgement}

The work was supported by the Coordination Center for Biomedical Technology of the University of Twente. The authors wish to thank Prof. W J Kolff (Utah University) for valuable advice. Thanks are due to Dr Rakhorst and C Dassel (University of Groningen) for the calf measurements.

\section{References}

De Mul F F M, Greve J and Hoeben F P Patcht application no 90019533 Septenter 1930

Wurzell D, Kolff J, Missfeldt W, Wildevuru W, Hansen G, Brownstcin L, Ricbman J B, DePaulis $\mathrm{R}$ and Kolff W J 1988 Development of thePhiladelphia heart system XIVth Congress of the European Society for Artificial Organs 